\title{
Nonparametric detector performance for active sonar in ocean reverberation background with multiple false targets jamming
}

\author{
Songwen Li \\ Shanghai marine electronic equipment research institute \\ 5200 Jindu road, Shanghai, 201108, China, \\ lisongwen@smmail.cn
}

\begin{abstract}
Passive sonar, which used to be the most effective device for submarine detection, cannot satisfy the requirement of anti-submarine operation after the appearance of quiet submarine decades ago, and active sonar is playing a more important role. Active sonar often works in reverberation limited condition in shallow water area because of the strong backscattering of the sea bottom. Unlike the volume reverberation often encountered by sonar working in deep ocean, bottom reverberation is usually not Rayleigh distributed. Experiment results show that the sea bottom reverberation is more likely to be $k$-distributed, thus the nonparametric detector, such as generalize sign detector, is more suitable for active sonar working in shallow water area. Multiple false targets can decrease the detection possibility of parametric and nonparametric CFAR detector, and usually used as an important method to counter active sonar. From Monte Carlo simulation, it is shown that only several false targets are needed to make the active sonar detection performance decline severely when it is using nonparametric detector in ocean reverberation background.
\end{abstract}

Index: nonparametric detector, multiple false targets, active sonar, ocean reverberation

\section{INTRODUCTION}

As early as world-war-II, active sonar had been the major device for submarine detection. But the frequency for active sonar at that time was usually larger than $20 \mathrm{kHz}$, that is, in ultrasonic frequency band. The detection range was limited because there is strong absorption for ultrasound propagates through sea water. After world-war-II, low frequency passive sonars with large aperture were developed in order to detect submarine from long distance by listening noise transmitted from submarine. Recently, with the appearance of quiet submarine, passive sonar cannot satisfy the anti-submarine operation anymore because of the significant reduction of detection range, and low frequency active sonar is becoming more and more important in submarine detection [1][2].

Reverberation is the most important interference for active sonar detection. For shallow water area, bottom reverberation is the major interference that limits the detection range of active sonar.

Almost all the modern sonar use multi-beam techniques and the data rate are much higher than the old single beam

\author{
Yang Song \\ Shanghai marine electronic equipment research institute \\ 5200 Jindu road, Shanghai, 201108, China \\ lisongwen@smmail.cn
}

sonar. It is almost impossible for sonar operator to find target only by observation and listening with such high data rate, and automatic detector is necessary for modern sonar [3]. The probability of detection for the automatic detector of active sonar is usually determined by signal-to-reverberation rate (for the following description in this paper, the SNR is used to represent signal-to-reverberation rate). Since the ocean bottom reverberation usually fluctuate severely, CFAR detector is necessary for active sonar in order to avoid high false alarm rate. It is reasonable to think if multiple man-made false targets are placed around a real target, the CFAR detector performance will decline significantly, and multiple false targets jamming can be an effective way of sonar countermeasure.

Generally, the ocean bottom reverberation is nonstationary with unknown distribution, it is proper to use nonparametric detector for active sonar to maintain CFAR. The nonparametric detector performance influenced by multiple false targets jamming in ocean bottom reverberation background is researched through Monte Carlo simulation in this paper.

\section{STATISTIC MODEL OF OCEAN REVERBERATION AND UNDERWATER ACOUSTIC TARGETS}

In early time ocean reverberation was often regarded as Rayleigh distributed, which is accurate in general if only ocean volume reverberation is considered when active sonar works in deep ocean. The ocean volume reverberation is caused by large amount of random micro-backscatterers within the sea water from far away, and can be regarded as Rayleigh distributed reverberation according to central limit theorem. But for sonar working in shallow water area, the bottom reverberation is dominating, and ocean bottom is usually un-flat with random strong backscattering structures, it is not proper to assume the bottom reverberation as Rayleigh distributed, especially for high resolution active sonar on account that the requirement of large amount of microbackscatterers for central limit theorem cannot be satisfied with too small bottom area enlightened by narrow sonar beam. The bottom reverberation is more likely to be $k$-distributed reverberation from the experiment data.

The pdf of k-distribution is[4] : 


$$
\begin{array}{r}
f_{X}(x \mid \alpha, \lambda)=\frac{4}{\sqrt{\lambda} \Gamma(\alpha)}\left(\frac{x}{\sqrt{\lambda}}\right)^{\alpha} \mathrm{K}_{\alpha-1}\left(\frac{2 x}{\sqrt{\lambda}}\right) \\
\sqrt{ } \quad \sqrt{ } \quad \sqrt{ }
\end{array}
$$

Where $\alpha$ is defined as shape parameter and $\lambda$ the scale parameter.

A simple way to generated $k$-distributed random variables is to use Gauss distributed and gamma distributed random variables. If $Z$ is a complex Gauss random variable with mean value of zero and variance of $\lambda, V$ is a gamma distributed variable independent from $Z$ with 1 as the scale parameter and $\alpha$ as the shape parameter, a $k$-distributed variable can be expressed as:

$$
X=\sqrt{V} Z
$$

The shape parameter and scale parameter for $X$ are $\alpha$ and $\lambda$, respectively.

Complex Gauss random variable is obtained from:

$$
Z=\sqrt{\lambda / 2}\left(W_{R}+j W_{I}\right)
$$

Where $W_{R}, W_{I}$ are independent Gauss random variable with mean value of zero and variance of 1 .

When $\alpha$ is a natural number, $V$ is obtained from:

$$
V=\sum_{i=1}^{\alpha}-\log U_{i}
$$

Here $U_{i}$ is a uniform distributed random variable between $(0,1)$.

$\alpha=1$ is chosen in the simulation to generate $k$-distributed reverberation.

Similar to radar target, sonar target is also fluctuating target. Swerling II target model can be used for echo of submarine [3], and can be generated from [5]:

$$
y=\left(\frac{1}{\ln 2} \ln \frac{1}{r}\right)^{1 / 2}
$$

Here $\mathrm{r}$ is a uniform distributed random variable between $(0,1)$.

\section{NONPARAMETRIC CFAR DETECTOR}

Both parametric and nonparametric detectors are used as CFAR detector for active sonar [3]. Parametric detector is often used when the background is stationary and with known distribution, which can be realized by energy integration followed by comparing with a certain threshold value. But for non-stationary and unknown distributed background, nonparametric detector is necessary in order to keep constant false alarm rate.

Most of the nonparametric detectors use rank test which comparing the signal amplitude from detection unit with that from nearby reference units. Generalized sign (GS) test detector [6] is a common detector in radar detection, its rank $R_{j}$ and test static $T_{R}$ are expressed as [5][6]:

$$
\begin{aligned}
& R_{j}=\sum_{j=1}^{N} u\left(x_{j 0}-x_{j i}\right) \\
& T_{R}=\sum_{j=1}^{M} R_{j}
\end{aligned}
$$

Here $u(x)$ is step function, $u(x)$ is 1 when $x \geqslant 0$, otherwise is $0 . x_{j 0}$ is the signal amplitude from the detection unit and $x_{\mathrm{ji}}$ is that from the each of the reference unit. $\mathrm{N}$ is the number of reference units for each sonar sweep, and $M$ is the sweep number. To simplify the detection scheme, rank quantization method is often used [3]: first, comparing rank $R_{j}$ with a threshold $\mathrm{L}$, if $R_{j}>\mathrm{L}$, output 1 ; otherwise output 0 . For the second threshold $\mathrm{T}$, if $T_{R}>\mathrm{T}$, it means target present; otherwise no target present. The false alarm rate for rank quantization method can be calculated through binomial distribution. For $\mathrm{N}=16, \mathrm{M}=8$, Table 1 shows some false alarm rate when first threshold $\mathrm{L}$ and second threshold $\mathrm{T}$ choose different values [3].

Table 1

\begin{tabular}{|} 
N=16, M=8, false alarm rate for different $\mathrm{L}$ and $\mathrm{T}$ \\
\hline $\mathrm{L}$ & $\mathrm{T}$ & $\mathrm{P}_{\mathrm{FA}}$ \\
\hline \multirow{3}{*}{13} & 5 & $6.1 \times 10^{-4}$ \\
\cline { 2 - 3 } & 6 & $3.6 \times 10^{-5}$ \\
\cline { 2 - 3 } & 7 & $9.4 \times 10^{-7}$ \\
\hline \multirow{3}{*}{14} & 5 & $6.0 \times 10^{-5}$ \\
\cline { 2 - 3 } & 6 & $2.2 \times 10^{-6}$ \\
\cline { 2 - 3 } & 7 & $3.6 \times 10^{-8}$ \\
\hline
\end{tabular}

IV Monte CARlo Simulation

Multiple false targets jamming is a common method in radar countermeasure. The influence of multiple false targets jamming on parametric detector has been studied in [7]. In this paper, the nonparametric detector performance influenced by multiple false targets for active sonar working in ocean bottom reverberation background is researched through Monte Carlo simulation.

The parameters for rank quantization method in the simulation are:

$$
\begin{array}{ll}
\mathrm{N}=16, & \mathrm{M}=8 \\
\mathrm{~L}=14, & \mathrm{~T}=6
\end{array}
$$

From Table 1, the false alarm rate is $2.2 \times 10^{-6}$.

In the simulation, a series of complex data combined with reverberation, real target signal and multiple false targets signals was generated. The target signal and false targets signals are summarized with random phase angles and the false targets are set in the reference units randomly. Swerling II target model is used for all the real and false targets. As comparisons, non-fluctuating targets model are also used for both the real and false targets and for false targets only in the simulation.

The simulation treats the bottom reverberation background as $k$-distributed. But simulation results using Rayleigh distributed reverberation background are also given as comparisons.

Figure 1 6 show the calculated ROC curves. 


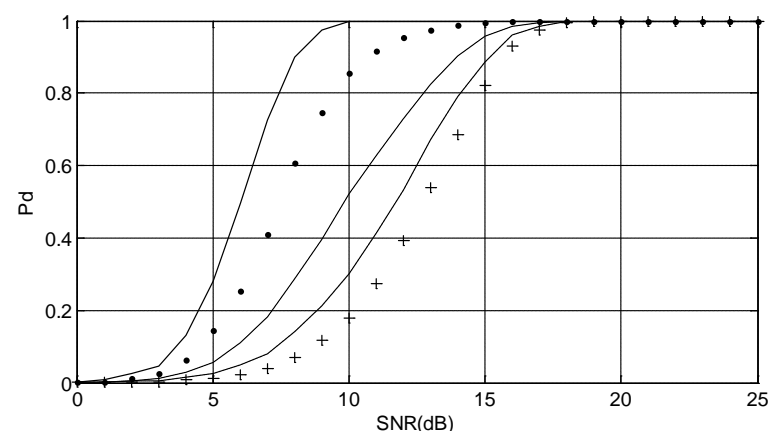

Fig. 1 curves from left to right represent detection probability in the condition of Rayleigh distributed reverberation background with 2, 4, 6, and 8 false targets jamming, respectively. Real and false targets are all assumed to be non-fluctuating. The false targets signal strength is $3 \mathrm{~dB}$ lower than the real targets signal.

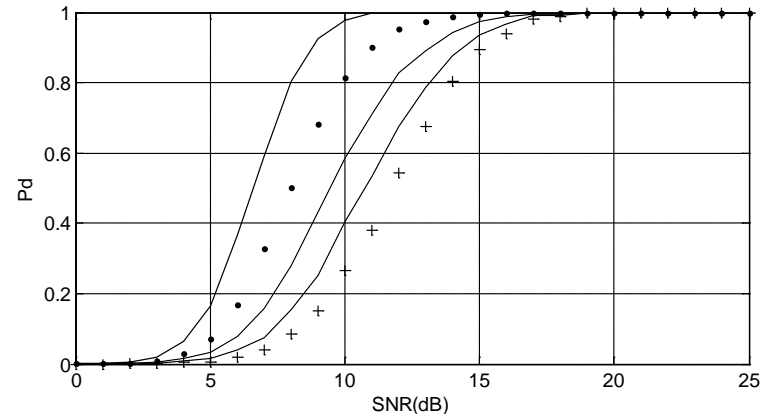

Fig. 2 curves from left to right represent detection probability in the condition of k-distributed reverberation background with 2, 4, 6 , and 8 false targets jamming, respectively. Real and false targets are all assumed to be non-fluctuating. The false targets signal strength is $3 \mathrm{~dB}$ lower than the real targets signal.

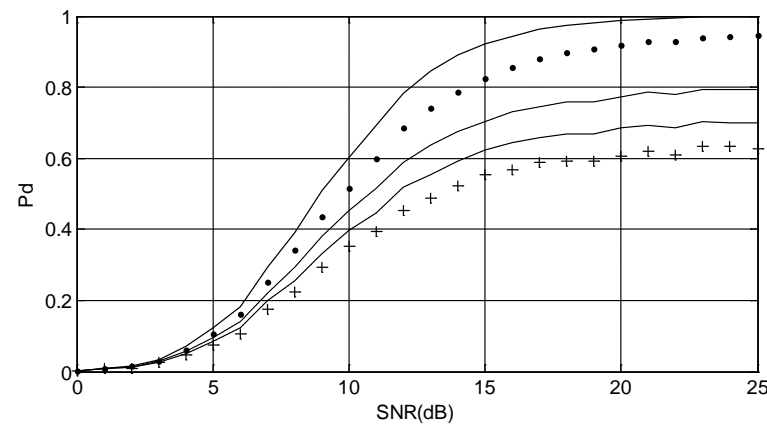

Fig. 3 curves from left to right represent detection probability in the condition of k-distributed reverberation background with 2, 4, 6 , and 8 false targets jamming, respectively. Swerling II target model is used for real and false targets. The signal strength of false targets is $10 \mathrm{~dB}$ lower than that of the real targets.

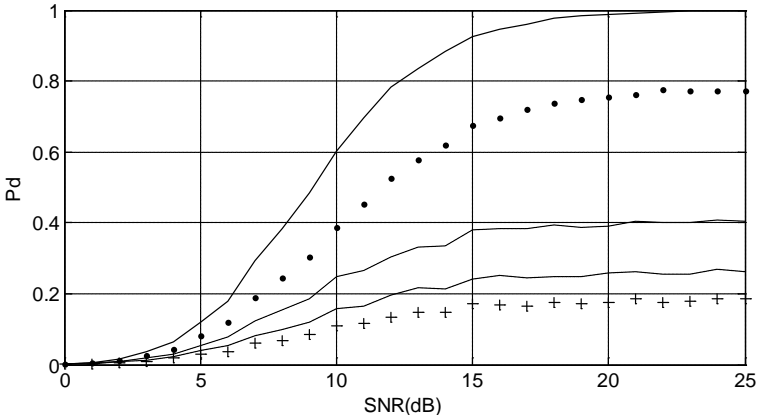

Fig. 4 curves from left to right represent detection probability in the condition of k-distributed reverberation background with 2, 4, 6 , and 8 false targets jamming, respectively. Swerling II target model is used for real and false targets. The signal strength of false targets is $6 \mathrm{~dB}$ lower than that of the real targets.

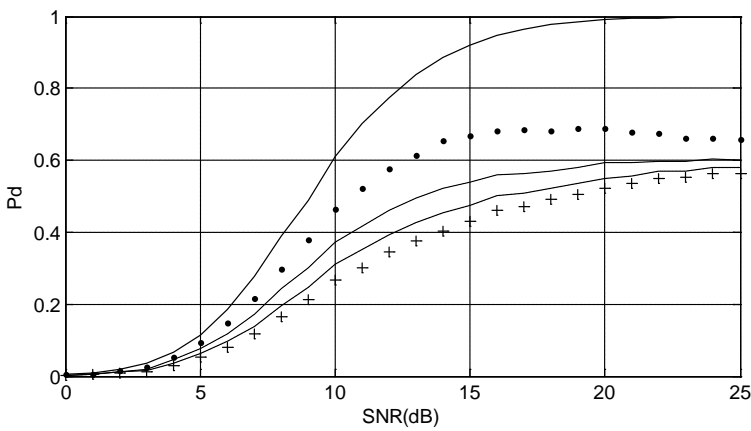

Fig. 5 curves from left to right represent detection probability in the condition of k-distributed reverberation background with 2, 4, 6 , and 8 false targets jamming, respectively. Swerling II target model is used for real target but non-fluctuating target model is used for false targets. The signal strength of false targets is $6 \mathrm{~dB}$ lower than that of the real targets.

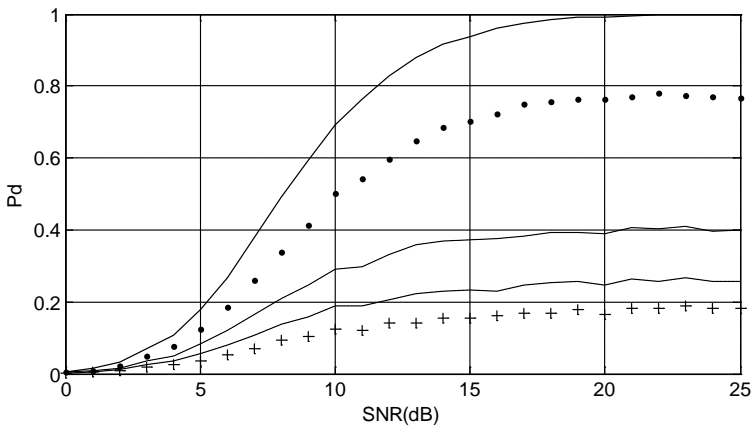

Fig. 6 curves from left to right represent detection probability in the condition of Rayleigh distributed reverberation background with 2, 4, 6, and 8 false targets jamming, respectively. Swerling II target model is used for real and false targets. The signal strength of false targets is $6 \mathrm{~dB}$ lower than that of the real targets. 


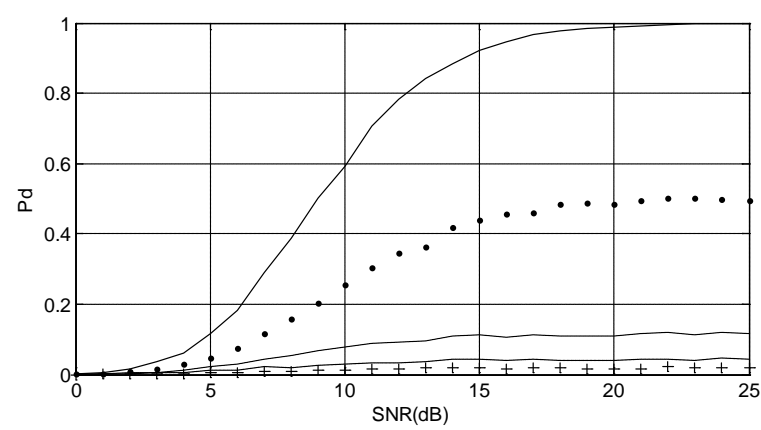

Fig. 7 curves from left to right represent detection probability in the condition of $k$-distributed reverberation background with 2, 4, 6 , and 8 false targets jamming, respectively. Swerling II target model is used for real and false targets. The signal strength of false targets is $3 \mathrm{~dB}$ lower than that of the real targets

Figure 1 and Fig. 2 show the results using non-fluctuating model for all the targets but with Rayleigh and $k$-distributed reverberation background respectively. It is shown that there is no significant difference for the detection performance between the two reverberation distribution models. To achieve $50 \%$ detection probability, the SNR requirement in $k$ distributed reverberation background is only about $1 \mathrm{~dB}$ higher than that in Rayleigh distributed reverberation background. The detection performance is not influenced severely by the multiple false targets jamming when nonfluctuating target mode is used: even there are 8 false targets within the 16 whole reference units with the target strength of each false target 3dB lower than that of the real target, SNR of $12 \mathrm{~dB}$ is enough to achieve $50 \%$ detection probability, only $5 \mathrm{~dB}$ higher than no false target jamming condition.

But when the targets strength is fluctuating, the detection performance will decline significantly by multiple false targets jamming. Fig. 3 shows that even the targets strength of each false target is $10 \mathrm{~dB}$ lower than that of the real target, 8 false targets jamming will increase the SNR requirement to $14 \mathrm{~dB}$ to achieve $50 \%$ detection probability. Fig. 4 shows when the target strength of each false target increased to $6 \mathrm{~dB}$ lower than the real target strength, only 2 false targets jamming will make the SNR requirement to $13 \mathrm{~dB}$ to achieve $50 \%$ detection probability, and 4 or more false targets jamming will make the $50 \%$ detection probability unreachable. Fig. 7 shows when the target strength of each false target increased to $3 \mathrm{~dB}$ lower than the real target strength, only 2 false targets jamming will make the SNR requirement to above $20 \mathrm{~dB}$ to achieve $50 \%$ detection probability

It is worth noting that when the target strength of each false target is relatively large (e.g. $6 \mathrm{~dB}$ or $3 \mathrm{~dB}$ lower than the real target strength) the detection probability will not reach $100 \%$ gradually with the SNR increasing continuously as normal ROC curve do. It is because that when SNR is larger enough, the influence of detection performance by the reverberation background will tend to be zero and the detection performance is determined only by multiple false targets jamming, on account that the rank quantization method only compares the real target signal amplitude with each reference signal amplitude independently, and the reference signals are determined only by the false targets signals when SNR is high enough.

Figure 5 shows the ROCs when Swerling II target model is used for real target and non-fluctuating target model is used for false targets. Comparing Fig. 5 with Fig. 7, it is shown that when there are 4 or more false targets jamming, nonfluctuating target model causes weaker influence to the detection performance than Swerling II target model does. The explanation is that when Swerling II target model is used for false targets, the target strength for each false target will fluctuate independently. If the false target number is more than 4 , the probability for the target strengths of two false targets to be stronger than normal ones will larger than $50 \%$, this will increase the probability of $R_{j}$ to be 0 because $R_{j}$ is 0 for $\mathrm{N}=16$ and $\mathrm{L}=14$ if there are two reference signals lager than the real target signal.

From Fig. 4 and Fig. 6, it is shown that there is no significant difference for the detection performance between the Rayleigh distributed and $k$-distributed reverberation background when the Swerling II target model is used for all the real and false targets, the same as when non-fluctuating model is used.

\section{SUMMARY}

Unlike ocean volume reverberation, bottom reverberation cannot be modeled as Rayleigh distributed reverberation, and $k$-distributed reverberation model is more suitable. Monte Carlo simulation results show that the distribution of the reverberation background is not a very important element for nonparametric detector performance. No matter the reverberation is Rayleigh distributed or $k$-distributed, multiple false targets jamming will decrease the detection probability severely and is an effective way for sonar countermeasure.

\section{REFERENCES}

[1] GORDON D. TYLER, JR., “THE EMERGENCE OF LOWFREQUENCY ACTIVE ACOUSTICS AS A CRITICAL ANTISUBMARINE WARFARE TECHNOLOGY”, Johns Hopkins APL Technical Digest, 42-159, 13(1), 1992

[2] Guanling Liu et.al., Review and prospect of active sonar detection techniques, Technical Acoustics, 335-340, 26(2), 2007, in Chinese

[3] Ziqiang Hou and Guibin Li, Sonar signal processing-Principle and devices, Ocean press, Beijing, 1986, in Chinese

[4] D. A. Abraham, "Signal excess in K -distributed reverberation," IEEE J. Ocean. Eng., 526-536, 28(3), 2003.

[5] Zaigen Fang, A Monte Carlo simulation of Non-parametric radar detection, Journal of Beijing Industry Institute, 49 90, No.1, 1982, in Chinese

[6] Hansem, V.G., et al., Nonparametric radar extraction using a generalized sign test, IEEE Trans., 942-950, Vol.AES-7, 1970

[7] Wei Liu and Huaijun Zhou, Radar detection performance influenced by jamming of multi-false targets, Modern defense technique, 137-142, 37(4), 2009, in Chinese 